TM-2241

\title{
DISTRIBUTION OF INCOHERENT SPACE-CHARGE TUNE SHIFT OF A BI-GAUSSIAN BEAM
}

\author{
K.Y. Ng \\ Fermi National Accelerator Laboratory,* P.O. Box 500, Batavia, IL 60510
}

(April 15, 2004)

\begin{abstract}
The incoherent space-charge self-force tune shift of a bi-Gaussian coasting beam is revisited. The distribution density of the incoherent horizontal or vertical space-charge tune shift is computed numerically using a statistical method. Analytic approach has also been made with approximation. The results show a broad peak centered about 0.633 with a rms spread 0.168 of the linear space-charge tune shift.
\end{abstract}

* Operated by the Universities Research Association, Inc., under contract with the U.S. Department of Energy. 


\section{INTRODUCTION}

The incoherent space-charge self-force tune shift is an important parameter in the design of low-energy accelerators. Although it has been shown that the spread of this incoherent tune shift plays no direct bearing on parametric resonances, however, if it becomes large enough, the coherent space-charge tune shift will land the betatron tune onto a parametric resonant line resulting in the instability of the particle beam. $[1,2,3]$ The incoherent spacecharge tune shift will also shift the incoherent tune spread away from the coherent betatron tune line, leaving behind no Landau damping. [4, 5] To provide Landau damping to the coherent betatron growth, the required tune spread is usually supplied by sextupoles or octupoles, and the spread must be large enough to counteract the incoherent space-charge tune shift so that the coherent betatron line will be covered. To understand the amount of Landau damping tune spread required, it is important to understand the distribution of the incoherent space-charge tune shift. In this paper, we are going to study the incoherent spacecharge tune shift of a particle inside a coasting beam with transverse bi-Gaussian distribution. The average incoherent tune shift and the rms incoherent tune shift are computed. Finally, an approximate but analytic solution of the distribution density of incoherent tune shift is derived.

\section{SMALL-AMPLITUDE TUNE SHIFTS}

For simplicity, we consider a round beam with the same bare tune $\nu_{0}$ in both the $x$ and $y$-directions. The Hamiltonian describing the transverse motion of a particle inside the round coasting beam is

$$
H=\frac{p_{x}^{2}+p_{y}^{2}}{2}+\frac{\nu_{0}^{2}}{R^{2}} \frac{x^{2}+y^{2}}{2}-V_{s c}(r)
$$

where $R$ is the main radius of the accelerator ring. The space-charge potential of the transverse bi-Gaussian distributed beam is given by

$$
V_{s c}(r)=\int_{0}^{r} f_{\mathrm{sc}}\left(r^{\prime}\right) d r^{\prime},
$$

where the space-charge force at the distance $r=\sqrt{x^{2}+y^{2}}$ in the radial direction can be written as

$$
f_{\mathrm{sc}}(r)=\frac{e^{2} \lambda}{2 \pi \sigma^{2} \gamma^{3} m v^{2}} \frac{1}{2 \pi \epsilon_{0} r} \int_{0}^{r} e^{-r^{\prime 2} /\left(2 \sigma^{2}\right)} 2 \pi r^{\prime} d r^{\prime}
$$


In above, $\epsilon_{0}$ is the electric permittivity of free space. The beam has transverse rms spread $\sigma$ and longitudinal linear density $\lambda$. The beam particle has charge $e$, mass $m$, velocity $v=\beta c$, and energy $\gamma m c^{2}$, where $c$ is the velocity of light. The independent variable is $s$, the distance measured along the accelerator ring.

To derive the betatron tune in the presence of the space-charge force, it is best to go to action-angle variables. ${ }^{\dagger}$ Since the space-charge force is usually much smaller than the external focusing force, the space-charge potential will be treated as a perturbation.

Solution of the unperturbed horizontal motion can be written as ${ }^{\ddagger}$

$$
\left\{\begin{aligned}
x & =\sqrt{2 J_{x} \bar{\beta}} \cos \psi_{x}, \\
p_{x} & =-\sqrt{\frac{2 J_{x}}{\bar{\beta}}} \sin \psi_{x}
\end{aligned}\right.
$$

where $J_{x}$ and $\psi_{x}$ are the horizontal action-angle variables. Here, we have introduced the average betatron function $\bar{\beta}=R / \nu_{0}$. Since $p_{x}=d x / d s$, we find that $d \psi / d s=1 / \bar{\beta}$ is also satisfied. Including the vertical components, the unperturbed Hamiltonian is therefore

$$
H_{0}=\frac{p_{x}^{2}+p_{y}^{2}}{2}+\frac{\nu_{0}^{2}}{R^{2}} \frac{x^{2}+y^{2}}{2}=\frac{\nu_{0}}{R}\left(J_{x}+J_{y}\right)
$$

From the Hamilton equations of motion,

$$
\frac{d \psi_{x, y}}{d s}=\frac{\partial H}{\partial J_{x, y}}
$$

${ }^{\dagger}$ The author would like to thank Dr. A. Burov for reminding him to use action-angle variables in deriving the space-charge tune shift.

† The canonical transformation can be derived from the generating function

$$
F_{1}\left(x, \psi_{x}\right)=\int_{0}^{x} p_{x} d x=-\frac{x^{2}}{2 \bar{\beta}} \tan \psi_{x}
$$

where

$$
p_{x}=-\frac{x}{\bar{\beta}} \tan \psi_{x},
$$

obtained from Eqs. (2.4) by eliminating $J_{x}$, has been substituted. The canonical transformation is then given by the above expression for $p_{x}$ and

$$
J_{x}=-\frac{\partial F_{1}}{\partial \psi_{x}}=\frac{x^{2}}{2 \bar{\beta}} \sec ^{2} \psi_{x}=\frac{x^{2}}{2 \bar{\beta}}\left(1+\frac{\bar{\beta}^{2} p_{x}^{2}}{x^{2}}\right)=\bar{\beta} H_{0} .
$$


the betatron tunes including space-charge effect can be conveniently derived:

$$
\frac{\nu_{x, y}}{R}=\frac{\partial\langle H\rangle}{\partial J_{x, y}},
$$

where $\langle H\rangle$ is the Hamiltonian averaged over the angle variables $\psi_{x}$ and $\psi_{y}$. This derivation is correct because the betatron tune is defined as the average number of betatron oscillations per revolution turn in the accelerator.

First let us derive the small-amplitude tunes. For this, the space-charge potential is linearized to obtain

$$
\Delta H=-V_{s c}(r)=-\frac{e^{2} \lambda r^{2}}{8 \pi \epsilon_{0} \gamma^{3} m v^{2} \sigma^{2}} .
$$

Replacing $r^{2}$ by the action-angle variables and in terms of the classical particle radius

$$
r_{0}=\frac{e^{2}}{4 \pi \epsilon_{0} m c^{2}},
$$

the linearized space-charge potential becomes

$$
\Delta H=-\frac{r_{0} \lambda R}{2 \nu_{0} \gamma^{3} \beta^{2} \sigma^{2}}\left(2 J_{x} \cos ^{2} \psi_{x}+2 J_{y} \cos ^{2} \psi_{y}\right)
$$

After averaging over the angle variables, the space-charge tune shifts are obtained according to Eq. (2.7),

$$
\Delta \nu_{x, y}=-\frac{r_{0} \lambda R^{2}}{2 \nu_{0} \gamma^{3} \beta^{2} \sigma^{2}}
$$

We denote these small-amplitude tune shifts by $\Delta \nu_{s c}^{\max }$, because these are the largest spacecharge tune shifts the particle experiences since the particle is seeing the largest space-charge force at the center of the beam. Because the linearized space-charge potential has been used, $\Delta \nu_{s c}^{\max }$ is also known as the linearized incoherent space-charge tune shift.

\section{LARGE-AMPLITUDE TUNE SHIFTS}

In terms of the small-amplitude space-charge tune shift $\Delta \nu_{s c}^{\max }$, the space-charge potential of Eq. (2.2) becomes

$$
\Delta H=-\Delta V_{s c}(r)=-\int_{0}^{r} d r^{\prime} \frac{4 \nu_{0}\left|\Delta \nu_{s c}^{\max }\right| \sigma^{2}}{R^{2} r^{\prime}}\left[1-e^{-r^{\prime 2} /\left(2 \sigma^{2}\right)}\right],
$$

where

$$
r^{2}=x^{2}+y^{2}=2 J_{x} \bar{\beta} \cos ^{2} \psi_{x}+2 J_{y} \bar{\beta} \cos ^{2} \psi_{y} .
$$


The tune shift in the $x$-direction is given by

$$
\frac{\Delta \nu_{x}}{R}=-\frac{\partial}{\partial J_{x}}\left\langle\Delta V_{s c}(r)\right\rangle c=-\left\langle\frac{\bar{\beta} \cos ^{2} \psi_{x}}{r} \frac{d}{d r} \Delta V_{s c}(r)\right\rangle,
$$

where $\langle\cdots\rangle$ implies averaging over the angle variables. We notice from Eq. (3.1) that the dependence on $J_{x}$ is through $r$ only, which is the upper limit of the integral. This leads to the last term in Eq. (3.3), which reduces to

$$
\frac{\Delta \nu_{x}}{\Delta \nu_{s c}^{\max }}=\left\langle\frac{2 \cos ^{2} \psi_{x}}{r^{2} /\left(2 \sigma^{2}\right)}\left[1-e^{-r^{2} /\left(2 \sigma^{2}\right)}\right]\right\rangle,
$$

or

$$
\frac{\Delta \nu_{x}}{\Delta \nu_{s c}^{\max }}=\int_{0}^{2 \pi} \frac{d \psi_{x}}{2 \pi} \int_{0}^{2 \pi} \frac{d \psi_{y}}{2 \pi} 2 \cos ^{2} \psi_{x} \frac{1-\exp \left[-\frac{J_{x}}{\sigma^{2} / \bar{\beta}} \cos ^{2} \psi_{x}-\frac{J_{y}}{\sigma^{2} / \bar{\beta}} \cos ^{2} \psi_{y}\right]}{\frac{J_{x}}{\sigma^{2} / \bar{\beta}} \cos ^{2} \psi_{x}+\frac{J_{y}}{\sigma^{2} / \bar{\beta}} \cos ^{2} \psi_{y}}
$$

giving the expression for the horizontal space-charge tune shift of a particle with horizontal and vertical amplitudes $\hat{x}=\sqrt{2 J_{x} \bar{\beta}}$ and $\hat{y}=\sqrt{2 J_{y} \bar{\beta}}$.

\section{TUNE SHIFT DISTRIBUTION}

To study the distribution in space-charge tune shift, first we must write down the distribution of the transverse particle offset in the beam. Let us concentrate on the distribution of the horizontal offset. Since the distribution density must be a function of the Hamiltonian, a Gaussian distribution density can be written as

$$
f\left(H_{0}\right)=\frac{1}{2 \pi\left(\sigma^{2} / \bar{\beta}\right)} e^{-\bar{\beta}^{2} H_{0} / \sigma^{2}}=\frac{1}{2 \pi\left(\sigma^{2} / \bar{\beta}\right)} e^{-\left(x^{2}+\bar{\beta}^{2} p_{x}^{2}\right) /\left(2 \sigma^{2}\right)},
$$

which is normalized to unity via integration over $x$ and $p_{x}$. Since we are after the distribution in amplitude, we need to go to action-angle variables, or

$$
f\left(H_{0}\right)=\frac{1}{2 \pi\left(\sigma^{2} / \bar{\beta}\right)} e^{-J_{x} /\left(\sigma^{2} / \bar{\beta}\right)} .
$$

The distribution density of $J_{x}$ is obtained by integration over $\psi_{x}$. Including the vertical components, we arrive at the distribution density

$$
f\left(J_{x}, J_{y}\right)=\frac{e^{-\left(J_{x}+J_{y}\right) /\left(\sigma^{2} / \bar{\beta}\right)}}{\left(\sigma^{2} / \bar{\beta}\right)^{2}} .
$$


The average horizontal space-charge tune shift is then written as

$$
\left\langle\frac{\Delta \nu_{x}}{\Delta \nu_{s c}^{\max }}\right\rangle=\int_{0}^{\infty} d J_{x} \int_{0}^{\infty} d J_{y} \frac{e^{-\left(J_{x}+J_{y}\right) /\left(\sigma^{2} / \bar{\beta}\right)}}{\left(\sigma^{2} / \bar{\beta}\right)^{2}} \frac{\Delta \nu_{x}}{\Delta \nu_{s c}^{\max }},
$$

with $\Delta \nu_{x} / \Delta \nu_{s c}^{\max }$ given by Eq. (3.5). The second moment is given by

$$
\left\langle\left(\frac{\Delta \nu_{x}}{\Delta \nu_{s c}^{\max }}\right)^{2}\right\rangle=\int_{0}^{\infty} d J_{x} \int_{0}^{\infty} d J_{y} \frac{e^{-\left(J_{x}+J_{y}\right) /\left(\sigma^{2} / \bar{\beta}\right)}}{\left(\sigma^{2} / \bar{\beta}\right)^{2}}\left(\frac{\Delta \nu_{x}}{\Delta \nu_{s c}^{\max }}\right)^{2},
$$

and the rms incoherent horizontal space-charge tune spread is

$$
\left(\frac{\Delta \nu_{x}}{\Delta \nu_{s c}^{\max }}\right)_{\mathrm{rms}}=\sqrt{\left\langle\left(\frac{\Delta \nu_{x}}{\Delta \nu_{s c}^{\max }}\right)^{2}\right\rangle-\left\langle\frac{\Delta \nu_{x}}{\Delta \nu_{s c}^{\max }}\right\rangle^{2}} .
$$

Unfortunately, the integrals in Eqs. (4.4) and (4.5) cannot be performed analytically, and they cannot even be simplified further. However, numerical evaluation is quite simple, resulting in ${ }^{\S}$

$$
\left\langle\frac{\Delta \nu_{x}}{\Delta \nu_{s c}^{\max }}\right\rangle=0.6334 \text { and } \quad\left(\frac{\Delta \nu_{x}}{\Delta \nu_{s c}^{\max }}\right)_{\mathrm{rms}}=0.1678
$$

It is nontrivial to derive the distribution of the space-charge tune shift in either the horizontal or vertical direction. This is because $\Delta \nu_{x}$ is a function of two variables $J_{x}$ and $J_{y}$. We therefore need to follow a method to convert two variables to one. Actually, $\Delta \nu_{x}$ is dependent on $J_{x}$ and $J_{y}$ through one variable which we denote by $u\left(J_{x}, J_{y}\right)$ and is independent of the other orthogonal combination $v\left(J_{x}, J_{y}\right)$. After integrating over $v$, we obtain the distribution of $u$, which can be transformed to the distribution of $\Delta \nu_{x}$ via a Jacobian. Unfortunately, we do not know of a way to derive $u\left(J_{x}, J_{y}\right)$, because Eq. (3.5) cannot be integrated analytically.

Nevertheless, the distribution of the space-charge tune shift can be obtained numerically using a statistical method. Ten millions particles are populated in the beam cross section at random according to the bi-Gaussian distribution. The space-charge tune shift of each of these particles can be computed using Eq. (3.5) or more easily using Eq. (6.6) below. The tune-shift distribution is then derived by putting the particles into 1000 bins of equal width so that the fractional $\mathrm{rms}$ statistical fluctuation is only $1 \%$. The result is plotted in Fig. 1. Alongside is an analytic approximate in dashes to be discussed in the next section. We see that distribution density starts from zero at the maximum tune shift $\Delta \nu_{s c}^{\max }$. This is expected, because only those few particles at the center of the beam will have tune shift

\footnotetext{
$\S$ These results have been obtained before, for example, by A. Burov.
} 


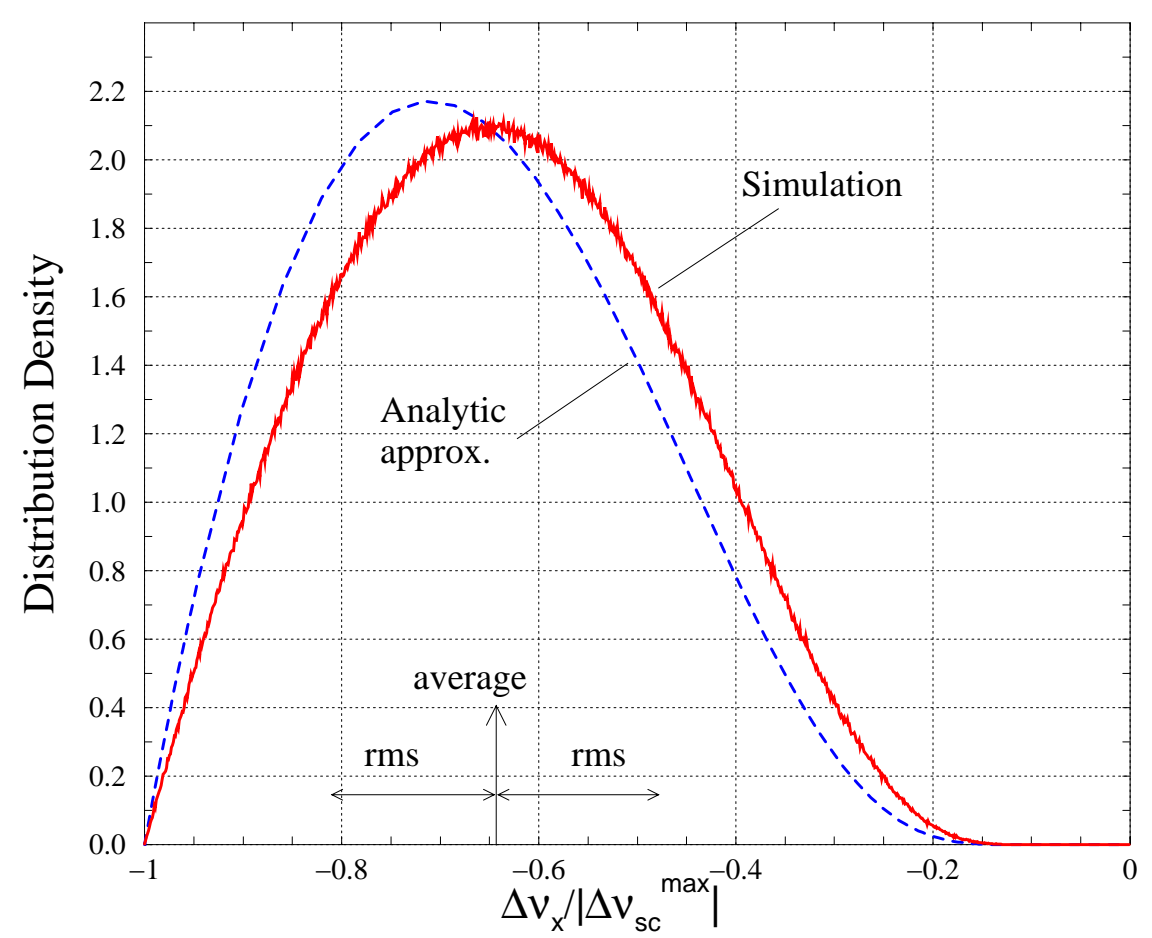

Figure 1: Distribution density of particles with incoherent horizontal space-charge tune shift $\Delta \nu_{x}$, in units of the maximum $\left|\Delta \nu_{s c}^{\max }\right|$. Shown in red is the numerical computation using ten million particles populated in the beam cross section according to the bi-Gaussian distribution. The average and rms spread of the distribution are marked. Shown in the dashed blue curve is the result of an analytic approximate derived in Sec. 5.

equal to $\Delta \nu_{s c}^{\max }$. The distribution is skew with a peak near $\Delta \nu_{x} /\left|\Delta \nu_{s c}^{\max }\right| \sim-0.65$, although the average tune shift is 0.63 of $\Delta \nu_{s c}^{\max }$. This distribution of incoherent tune shift can now be employed in the dispersion relation to determine whether a certain transverse beam dynamic effect can be Landau-stabilized or not.

\section{ANALYTIC APPROXIMATE}

To pursue an analytic solution, the averaging over the angle variables in Eq. (3.5) is approximated through the substitution of cosine square by $\frac{1}{2}$. We obtain immediately the approximate result

$$
\frac{\Delta \nu_{x}}{\Delta \nu_{m}} \approx \frac{1-\exp \left[-\frac{J_{x}}{2 \sigma^{2} / \beta}-\frac{J_{y}}{2 \sigma^{2} / \beta}\right]}{\frac{J_{x}}{2 \sigma^{2} / \beta}+\frac{J_{y}}{2 \sigma^{2} / \beta}}
$$


In order to check how good this approximation is, this analytic expression is substituted into Eqs. (4.4) and (4.5) to compute the first and second moments of the horizontal space-charge tune shift. The integrals are easy to perform now, giving the results

$$
\left\langle\frac{\Delta \nu_{x}}{\Delta \nu_{s c}^{\max }}\right\rangle=\frac{2}{3}=0.6667 \text { and }\left(\frac{\Delta \nu_{x}}{\Delta \nu_{s c}^{\max }}\right)_{\mathrm{rms}}=\sqrt{4 \ln \frac{9}{8}-\left(\frac{2}{3}\right)^{2}}=0.1634
$$

which are very close to the numerical results depicted in Eq. (4.7). As another test of the approximation, let us compute the horizontal space-charge tune shift as a function of the horizontal amplitude $\hat{x}=\sqrt{2 \bar{\beta} J_{x}}$ regardless of what the vertical amplitude $\hat{y}=\sqrt{2 \beta J_{y}}$ is. This implies the integration of $\Delta \nu_{x}$ over $J_{y}$ with the particle distribution included. With the analytic expression of Eq. (5.9), this tune shift is given by

$$
\left.\frac{\Delta \nu_{x}}{\Delta \nu_{s c}^{\max }}\right|_{\text {any } J_{y}}=\int_{0}^{\infty} d t_{y} e^{-t_{y}} \frac{1-e^{-\left(t_{x}+t_{y}\right) / 2}}{\left(t_{x}+t_{y}\right) / 2}
$$

where we have substituted $t_{x}=J_{x} \bar{\beta} / \sigma^{2}=\hat{x}^{2} /\left(2 \sigma^{2}\right)$ and $t_{y}=J_{y} \bar{\beta} / \sigma^{2}=\hat{y}^{2} /\left(2 \sigma^{2}\right)$. The result is shown as a dashed curve in Fig. 2. In the same figure, we also show the exact numerically derived solution as a solid curve. Although the two curves differ, however, the difference is not too much, implying that the approximation is acceptable. We see in the figure that the horizontal tune shift at zero horizontal amplitude is only 0.828 (0.811 for the approximated solution) of the maximum tune shift $\Delta \nu_{s c}^{\max }$. This is not unexpected, because only the particle at zero vertical offset $(y=0)$ has the horizontal tune shift $\Delta \nu_{s c}^{\max }$ when the horizontal amplitude is infinitesimal. With a nonvanishing vertical betatron amplitude, the particle spends part of the time at a vertical offset $(y \neq 0)$. Since the particle does not pass through the center of the bunch, it is seeing less space-charge force and therefore experiencing a smaller space-charge tune shift.

To find the distribution as a function of tune shift, let us go back to Eq. (5.8), which is the approximated analytic expression of Eq. (3.5). Although the tune shift depends on both the horizontal and vertical amplitudes, $J_{x}$ and $J_{y}$, its dependency in the approximated expression is actually $J_{x}+J_{y}$ or $u=\left(t_{x}+t_{y}\right) / 2$. The distribution density we are looking for becomes very simple now. First, from the distribution in amplitudes, $f\left(J_{x}, J_{y}\right)$ of Eq. (4.3), we integrate over $v=t_{x}-t_{y}$ to obtain the particle distribution in $u$,

$$
\int f\left(J_{x}, J_{y}\right) d t_{x} d t_{y}=\left[\int_{-2 u}^{2 u} d v e^{-u}\right] d u=4 u e^{-u} d u
$$

Next, the distribution in tune shift is obtained by a transformation from $u$ to $\Delta \nu_{x}$, resulting 


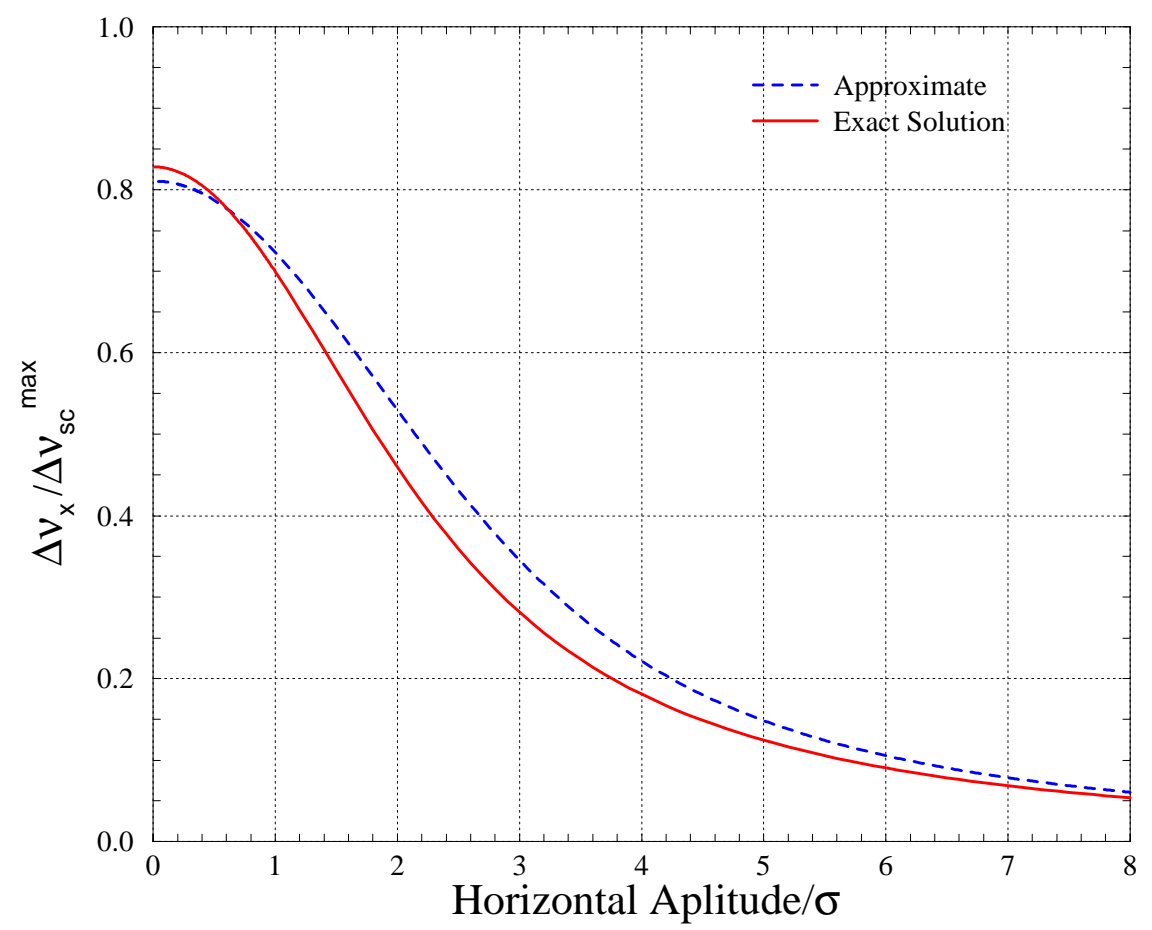

Figure 2: Incoherent horizontal space-charge tune shift $\Delta \nu_{x}$, in units of the maximum $\Delta \nu_{s c}^{\max }$, of a particle with horizontal betatron amplitude in units of rms beam size $\sigma$ inside a beam with bi-Gaussian distribution. The solid curve is the exact numerical solution and the dashed curve is the approximated analytical solution.

in

$$
f(u) d u=\left[4 u e^{-2 u} \frac{d u}{d \Delta \nu_{x}}\right] d \Delta \nu_{x}=f\left(\frac{\Delta \nu_{x}}{\Delta \nu_{s c}^{\max }}\right) d\left(\frac{\Delta \nu_{x}}{\Delta \nu_{s c}^{\max }}\right) .
$$

The distribution density of $\Delta \nu_{x}$ becomes

$$
f\left(\frac{\Delta \nu_{x}}{\Delta \nu_{s c}^{\max }}\right)=\frac{4 u^{3} e^{-2 u}}{1-(1+u) e^{-u}},
$$

where $u$ is considered as a function of $\Delta \nu_{x}$ through

$$
\frac{\Delta \nu_{x}}{\Delta \nu_{s c}^{\max }}=\frac{1-e^{-u}}{u}
$$

The result is depicted in Fig. 1 in dashes. We see that although the analytic approximate leans backward from the actual distribution by a few percents, however, it does has the same general shape as the actual distribution. 


\section{Note added in proof:}

We were informed by Dr. T. Sen that the average horizontal tune can be computed analytically, and our derivation here had been very similar to his derivation of beam-beam tune shift for two round beams colliding head-on. We include the analytic derivation below.

In the bi-Gaussian round beam model, the space-charge potential defined in Eq. (2.2) can be written as

$$
V_{s c}(r)=\frac{1}{\gamma^{3} m v^{2}} \frac{e^{2} \lambda}{4 \pi \epsilon_{0}} \int_{0}^{\infty} d t \frac{e^{-\left(x^{2}+y^{2}\right) /\left(2 \sigma^{2}+t\right)}}{2 \sigma^{2}+t} .
$$

The derivation is included in the appendix for completeness. The merit of this representation is that the variables $x$ and $y$ become separable. The implication is that the angle variables can be integrated. For example

$$
\begin{aligned}
\int_{0}^{2 \pi} e^{-x^{2} /\left(2 \sigma^{2}+t\right)} \frac{d \psi_{x}}{2 \pi} & =\int_{0}^{2 \pi} e^{-2 J_{x} \bar{\beta} \cos ^{2} \psi_{x} /\left(2 \sigma^{2}+t\right)} \frac{d \psi_{x}}{2 \pi} \\
& =e^{-J_{x} \bar{\beta} /\left(2 \sigma^{2}+t\right)} \int_{0}^{2 \pi} e^{-J_{x} \bar{\beta} \cos 2 \psi_{x} /\left(2 \sigma^{2}+t\right)} \frac{d \psi_{x}}{2 \pi} \\
& =e^{-J_{x} \bar{\beta} /\left(2 \sigma^{2}+t\right)} I_{0}\left(\frac{J_{x} \bar{\beta}}{2 \sigma^{2}+t}\right)
\end{aligned}
$$

where $I_{n}$ is the modified Bessel function of order $n$. We therefore obtain the space-charge potential averaged over angle variables:

$$
\left\langle V_{s c}\left(J_{x}, J_{y}\right)\right\rangle=\frac{2 \nu_{0} \sigma^{2}\left|\Delta \nu_{s c}^{\max }\right|}{R^{2}} \int_{0}^{\infty} \frac{d t}{2 \sigma^{2}+t} I_{0}\left(\frac{J_{x} \bar{\beta}}{2 \sigma^{2}+t}\right) I_{0}\left(\frac{J_{y} \bar{\beta}}{2 \sigma^{2}+t}\right) e^{-\left(J_{x}+J_{y}\right) \bar{\beta} /\left(2 \sigma^{2}+t\right)},
$$

where $\Delta \nu_{s c}^{\max }$, the maximum space-charge tune shift of Eq. (2.11) has been substituted. The horizontal horizontal tune shift is given by taking partial derivative with respect to $J_{x}$, leading to

$$
\frac{\Delta \nu_{x}}{\left|\Delta \nu_{s c}^{\max }\right|}=\frac{2 \sigma^{2}}{\bar{\beta}} \int_{0}^{\infty} \frac{\bar{\beta} d t}{\left(2 \sigma^{2}+t\right)^{2}} e^{-\left(J_{x}+J_{y}\right) \bar{\beta} /\left(2 \sigma^{2}+t\right)} I_{0}\left(\frac{J_{y} \bar{\beta}}{2 \sigma^{2}+t}\right)\left[I_{0}\left(\frac{J_{x} \bar{\beta}}{2 \sigma^{2}+t}\right)-I_{1}\left(\frac{J_{x} \bar{\beta}}{2 \sigma^{2}+t}\right)\right] .
$$

With the introduction of the variables

$$
s_{x, y}=\frac{J_{x, y} \bar{\beta}}{\sigma^{2}} \quad \text { and } \quad z=\frac{2 \sigma^{2}}{2 \sigma^{2}+t}
$$

the above simplifies to

$$
\frac{\Delta \nu_{x}}{\left|\Delta \nu_{s c}^{\max }\right|}=\int_{0}^{1} d z e^{-\frac{1}{2} s_{x} z-\frac{1}{2} s_{y} z} I_{0}\left(\frac{1}{2} s_{y} z\right)\left[I_{0}\left(\frac{1}{2} s_{x} z\right)-I_{1}\left(\frac{1}{2} s_{x} z\right)\right] .
$$


Including the distribution in $J_{x}$ and $J_{y}$ of Eq. (4.3), the average horizontal space-charge tune shift becomes

$$
\frac{\left\langle\Delta \nu_{x}\right\rangle}{\left|\Delta \nu_{s c}^{\max }\right|}=\int_{0}^{1} d z \int_{0}^{\infty} d s_{x} \int_{0}^{\infty} d s_{y} e^{-s_{x}\left(1+\frac{1}{2} z\right)-s_{y}\left(1+\frac{1}{2} z\right)} I_{0}\left(\frac{1}{2} s_{y} z\right)\left[I_{0}\left(\frac{1}{2} s_{x} z\right)-I_{1}\left(\frac{1}{2} s_{x} z\right)\right] .
$$

Since the integrals over the action variables decoupled, we can integrate over $s_{x}$ and $s_{y}$ separately and finally do the integration over z. From Gradshteyn and Ryzhik (6.611.4), [10]

$$
\int_{0}^{\infty} e^{-\alpha x} I_{\nu}(\beta x) d x=\frac{\beta^{\nu}}{\sqrt{\alpha^{2}-\beta^{2}}\left(\alpha+\sqrt{\alpha^{2}-\beta^{2}}\right)^{\nu}}
$$

provided that $\operatorname{Re} \nu>-1$ and $\operatorname{Re} \alpha>|\mathcal{R} e \beta|$, we obtain

$$
\begin{gathered}
\int_{0}^{\infty} d s_{x} e^{-s_{x}\left(1+\frac{1}{2} z\right)}\left[I_{0}\left(\frac{1}{2} s_{x} z\right)-I_{1}\left(\frac{1}{2} s_{x} z\right)\right]=\frac{1}{\sqrt{1+z}}\left[1-\frac{z}{(1+\sqrt{1+z})^{2}}\right] \\
\int_{0}^{\infty} d s_{y} e^{-s_{y}\left(1+\frac{1}{2} z\right)} I_{0}\left(\frac{1}{2} s_{y} z\right)=\frac{1}{\sqrt{1+z}}
\end{gathered}
$$

Hence

$$
\frac{\left\langle\Delta \nu_{x}\right\rangle}{\left|\Delta \nu_{s c}^{\max }\right|}=\int_{0}^{1} \frac{d z}{1+z}\left[1-\frac{z}{(1+\sqrt{1+z})^{2}}\right]
$$

The integral of the first term in the squared brackets is $\ln 2$. For the second term, change the variable of integration to $u=1 /(1+\sqrt{1+z})$. Then

$$
\int_{0}^{1} d z \frac{z}{(1+z)(1+\sqrt{1+z})^{2}}=\int_{\frac{1}{\sqrt{2}+1}}^{\frac{1}{2}} d u \frac{2(1-2 u)}{u(1-u)}=\left.2 \ln u(1-u)\right|_{\frac{1}{\sqrt{2}+1}} ^{\frac{1}{2}}=2 \ln \frac{(1+\sqrt{2})^{2}}{4 \sqrt{2}}
$$

The average horizontal tune shift becomes

$$
\frac{\left\langle\Delta \nu_{x}\right\rangle}{\left|\Delta \nu_{s c}^{\max }\right|}=\ln 2-2 \ln \frac{(1+\sqrt{2})^{2}}{4 \sqrt{2}}=\ln \frac{64}{(1+\sqrt{2})^{4}}=0.633389
$$

which is what we obtained before through numerical integration. 


\section{APPENDIX}

We follow a derivation by K. Takayama. [6] Unlike the traditional derivation which relies on spatial symmetry [7, 8] or the complicated elliptical coordinates, [9] this derivation is valid for any charge distribution and the simple Cartesian coordinates are used.

The potential $\phi(\vec{r})$ generated by a charge distribution $N e \rho(\vec{r})$, with $\rho$ normalized to unity, satisfies the Poisson's equation

$$
\nabla^{2} \phi(\vec{r})=-\frac{N e \rho(\vec{r})}{\epsilon_{0}}
$$

The Green's function satisfying

$$
\nabla^{2} G\left(\vec{r}, \overrightarrow{r^{\prime}}\right)=-\delta\left(\vec{r}-\overrightarrow{r^{\prime}}\right)
$$

is

$$
G\left(\vec{r}, \overrightarrow{r^{\prime}}\right)=\frac{1}{4 \pi \mid \vec{r}-\overrightarrow{r^{\prime} \mid}}=\frac{1}{2 \pi^{3 / 2}} \int_{0}^{\infty} e^{-\left|\vec{r}-\vec{r}^{\prime}\right|^{2} q^{2}} d q
$$

In the integral form, the Green's function becomes separable in the coordinates $(x, y, z)$. For later application, we change the variable of integration to $t=1 / q$. The potential now takes the form

$$
\phi(\vec{r})=\frac{N e}{4 \pi \epsilon_{0} \pi^{1 / 2}} \int_{0}^{\infty} \frac{d t}{t^{3 / 2}} \iint_{-\infty}^{\infty} \int \overrightarrow{r^{\prime}} \rho\left(\overrightarrow{r^{\prime}}\right) e^{-\left|\vec{r}-\vec{r}^{\prime}\right|^{2} / t} .
$$

This representation has the merit that it is applicable to any charge distribution even if it does not possess any symmetry, and the integration will be separable in the coordinates $\left(x^{\prime}, y^{\prime}, z^{\prime}\right)$ provided that $\rho\left(\overrightarrow{r^{\prime}}\right)$ is separable.

Now for the tri-Gaussian distribution

$$
\rho(\vec{r})=\frac{1}{(2 \pi)^{3 / 2} \sigma_{x} \sigma_{y} \sigma_{z}} \exp \left(-\frac{x^{2}}{2 \sigma_{x}^{2}}-\frac{y^{2}}{2 \sigma_{y}^{2}}-\frac{z^{2}}{2 \sigma_{z}^{2}}\right),
$$

where $\sigma_{x}, \sigma_{y}$, and $\sigma_{z}$ are the standard deviations,

$$
\phi(\vec{r})=\frac{N e}{2^{7 / 2} \pi^{3} \epsilon_{0} \sigma_{x} \sigma_{y} \sigma_{z}} \int_{0}^{\infty} \frac{d t}{t^{3 / 2}} \iint_{-\infty}^{\infty} \int d \overrightarrow{r^{\prime}} \rho\left(\overrightarrow{r^{\prime}}\right) \exp \left(-\frac{\left|\vec{r}-\overrightarrow{r^{\prime}}\right|^{2}}{t}-\frac{x^{\prime 2}}{2 \sigma_{x}^{2}}-\frac{y^{\prime 2}}{2 \sigma_{y}^{2}}-\frac{z^{\prime 2}}{2 \sigma_{z}^{2}}\right) .
$$

The integration over $x^{\prime}, y^{\prime}$, and $z^{\prime}$ can be performed easily using

$$
\int_{-\infty}^{\infty} \exp \left[-\frac{\left(x-x^{\prime}\right)^{2}}{t}-\frac{x^{\prime 2}}{2 \sigma_{x}^{2}}\right]=\frac{\sqrt{2 \pi t} \sigma_{x}}{\sqrt{2 \sigma_{x}+t}} .
$$


The final result is

$$
\phi(\vec{r})=\frac{N e}{4 \pi \epsilon_{0} \pi^{1 / 2}} \int_{0}^{\infty} d t \frac{\exp \left(-\frac{x^{2}}{2 \sigma_{x}^{2}+t}-\frac{y^{2}}{2 \sigma_{y}^{2}+t}-\frac{z^{2}}{2 \sigma_{z}^{2}+t}\right)}{\left(2 \sigma_{x}^{2}+t\right)^{1 / 2}\left(2 \sigma_{y}^{2}+t\right)^{1 / 2}\left(2 \sigma_{z}^{2}+t\right)^{1 / 2}} .
$$

For a bi-Gaussian coasting beam of charge density

$$
N e \rho(\vec{r})=\frac{e \lambda}{2 \pi \sigma_{x} \sigma_{y}} \exp \left(-\frac{x^{2}}{2 \sigma_{x}^{2}}-\frac{y^{2}}{2 \sigma_{y}^{2}}\right)
$$

where $\lambda$ is the linear density, substitution into Eq. (A.4) and integration over $\overrightarrow{r^{\prime}}$ leads to the two-dimensional potential

$$
\phi(\vec{r})=\frac{e \lambda}{4 \pi \epsilon_{0}} \int_{0}^{\infty} d t \frac{\exp \left(-\frac{x^{2}}{2 \sigma_{x}^{2}+t}-\frac{y^{2}}{2 \sigma_{y}^{2}+t}\right)}{\left(2 \sigma_{x}^{2}+t\right)^{1 / 2}\left(2 \sigma_{y}^{2}+t\right)^{1 / 2}} .
$$




\section{References}

[1] F.J. Sacherer, Transverse Space-Charge Effects in Circular Accelerator, Ph.D. Thesis, 1968, UCRL-18454.

[2] R. Baartman, Betatron Resonances with Space Charge, Proceedings of Int. Workshop on Emittance in Circular Accelerators Nov. 1994, KEK, Japan, KEK report 95-7, p.273; R. Baartman, Betatron Resonances with Space Charge, Proceedings of Workshop on Space Charge Physics in High Intensity Hadron Rings, p.73, Ed. Luccio, A.U., and Weng, W.T., (Shelter Island, New York, May 4-7, 1998).

[3] S. Machida and M. Ikegami, Simulation of Space Charge Effects in a Synchrotron, Proceedings of Workshop on Space Charge Physics in High Intensity Hadron Rings, p.73, Ed. Luccio, A.U., and Weng, W.T., (Shelter Island, New York, May 4-7, 1998).

[4] Yu. Alexahin, J. Annala, A. Burov, P. Ivanov, V. Shiltsev, T. Sen, and C.Y. Tan, Tevatron Transverse Instability Studies, Talk presented by P. Ivanov at the First MidWest Accelerator Physics Collaboration Meeting, October 4-5, 2002, Fermilab.

[5] P. Ivanov, J. Annala, A. Burov, V. Lebedev, E. E.Lorman, V. Ranijbar, V. Scarpine, and V. Shiltsev, Head-Tail Instability at Tevatron, Proceedings of the 2003 Particle Accelerator Conference, Portland, Oregon, 2003.

[6] Ken Takayama, A New Method for Potential of a 3-Dimensional Non-uniform Charge Distribution, Fermilab Report FN-365, 1982.

[7] O.D. Kellog, Foundations of Potential Theory, Springer-Verlag, 1967, p.184.

[8] E. Weber, Electromagnetic Fields, Theory and Applications, Vol. 1, John Wiley \& Sons, Inc, 1960, p.414.

[9] B.W. Montague, Fourth-Order Coupling Resonances Excited by Space-Charge Forces in a Synchrotron, CERN/ISR/68-38, 1968.

[10] I.S. Gradshtern and I.M. Ryzhik, Table of Integrals, Series, and Products, Academic Press, 1965. 